\title{
掺氮金刚石电极性能及其氧化降解硝基苯研究
}

\author{
严世胜 ${ }^{1}$ ，彭鸿赝 ${ }^{1}$ ，赵志斌 ${ }^{1}$ ，潘孟美 ${ }^{1}$ ，羊大立 ${ }^{1}$ ，阿金华 ${ }^{1}$, \\ 叶国林 ${ }^{1}$, 王崇太 ${ }^{2}$, 郭心琒 ${ }^{2}$
}

(海南师范大学 1. 物理与电子工程学院, 2. 化学与化工学院, 海口 571158)

摘 要: 采用热阴极直流辉光等离子体化学气相沉积法制备亚微米晶氮掺杂金刚石膜(NDD), 采用 SEM 分析样品的 表面形貌，分别用 Hall 测试和循环伏安法测试氮掺杂金刚石电极的电学和电化学性能。实验结果表明，当氮气流量 低于 $30 \mathrm{sccm}$ 时, 膜的电导率随氮气流量的增大略有提高; 氮气流量继续增大则电导率迅速下降，电导率最大为 $5.091 \mathrm{~S} / \mathrm{cm}$ 。氮掺杂金刚石电极具有较好的伏安性能, 在酸性、中性和碱性介质中均具有较宽的电位窗口和较低的 背景电流。以硝基苯为目标污染物测试 NDD 材料作为阳极氧化降解的电催化性能。在 $0.1 \mathrm{~mol} / \mathrm{L} \mathrm{Na}_{2} \mathrm{SO}_{4}$ 溶液的支 持电解质中，以氮掺杂金刚石为阳极降解 $0.5 \mathrm{mmol} / \mathrm{L}$ 的硝基苯，反应时间 $300 \mathrm{~min}$ ，硝基苯的降解率达到 $94 \%$, 化 学需氧量(COD)去除率约 $68 \%$ 。

关 键 词: 热阴极直流辉光等离子体化学气相沉积; 掺氮亚微米晶金刚石电极; 电催化氧化; 硝基苯降解 中图分类号: O646 文献标识码: A

\section{Nitrogen-doped Diamond Electrode Property and Anodic Catalytic Degradation of Nitrobenzene}

YAN Shi-Sheng ${ }^{1}$, PENG Hong-Yan ${ }^{1}$, ZHAO Zhi-Bin ${ }^{1}$, PAN Meng-Mei ${ }^{1}$, YANG Da-Li ${ }^{1}$, A Jin-Hua ${ }^{1}$, YE Guo-Lin ${ }^{1}$, WANG Chong-Tai ${ }^{2}$, GUO Xin-Wei ${ }^{2}$

(1. School of Physics and Electronic Engineering, Hainan Normal University, Haikou 571158, China; 2. School of Chemistry and Chemical Engineering, Hainan Normal University, Haikou 571158, China)

\begin{abstract}
A series of nitrogen-doped diamond (NDD) film electrodes were synthesis by hot cathode direct current plasma chemical vapor deposition (HCDCPCVD) method with varied ratio $\mathrm{CH}_{4} / \mathrm{H}_{2} / \mathrm{N}_{2}$ gas mixture. Morphologies of diamond films were characterized by SEM. Electrical and electrochemical properties of nitrogen-doped diamond electrodes were characterized by Hall test and cyclic voltammetry. The results show that when the nitrogen flow rate is less than $30 \mathrm{sccm}$, the conductivity of the film increases slightly with the increase of the nitrogen flow rate. As the nitrogen flow rate continues to increase, the conductivity decreases rapidly, showing the maximum electroconductibility of $5.091 \mathrm{~S} / \mathrm{cm}$. The nitrogen-doped diamond electrode has good voltammetric performance with a wide potential window and a low background current in acidic, neutral and alkaline media. Properties of anodic oxidation degradation of nitrogen-doped diamond electrodes were tested using nitrobenzene as target pollutant. In the supporting electrolyte of $0.1 \mathrm{~mol} / \mathrm{L} \mathrm{Na}_{2} \mathrm{SO}_{4}$ solution, $0.5 \mathrm{mmol} / \mathrm{L}$ nitrobenzene is decomposed using the nitrogendoped diamond as anode. After reaction for $300 \mathrm{~min}$, degradation rate of the nitrobenzene reaches $94 \%$, and COD (Chemical Oxygen Demand) removal rate is about 68\%.
\end{abstract}

收稿日期：2017-06-08; 收到修改稿日期：2017-10-02

基金项目：国家自然科学基金(51262007); 海南省自然科学基金(617104,20165196)

National Natural Science Foundation of China (51262007); Natural Science Foundation of Hainan Province (617104, 20165196)

作者简介：严世胜(1972-), 男, 副教授. E-mail: 605840548@qq.com

通讯作者：彭鸿雁，教授. E-mail: mdjphy@163.com 
Key words: hot cathode direct current glow plasma chemical vapor deposition; nitrogen-doped submicron-crystalline diamond electrode; electrocatalytic oxidation; nitrobenzene degradation

掺杂金刚石膜具有较宽的电势窗口、极低的背 景电流、稳定的化学性质、在强酸强碱中不会被腐 蚀、吸附惰性等特点, 在有机污水处理、电化学分 析等领域有良好的应用前景 ${ }^{[1-5]}$ 。对掺杂金刚石膜的 研究主要集中在掺嗍金刚石膜上 ${ }^{[6-8]}$, 而对掺氮金 刚石膜的研究比较少见。

Pleskov 等 ${ }^{[9]}$ 首次报道掺氮纳米晶金刚石膜的电 化学性能, 他们采用微波等离子体化学气相沉积法 (MPCVD), 以 $\mathrm{H}_{2} / \mathrm{Ar} / \mathrm{CH}_{4} / \mathrm{N}_{2}$ 混合气体为原料, 制备 掺氮纳米金刚石膜，当 $\mathrm{N}_{2}$ 流量比例为 $0 \sim 25 \%$ 时，膜 的电阻率为 $10^{10} \sim 3.3 \times 10^{-3} \Omega \cdot \mathrm{cm}$, 在 $2.5 \mathrm{~mol} / \mathrm{L} \mathrm{H}_{2} \mathrm{SO}_{4}$ 溶液中测得电势窗口为 $3.8 \sim 2.1 \mathrm{~V}$; 在 $1 \mathrm{~mol} / \mathrm{L} \mathrm{H}_{2} \mathrm{SO}_{4}+$ $0.01 \mathrm{~mol} / \mathrm{L} \mathrm{K}_{3}\left[\mathrm{Fe}(\mathrm{CN})_{6}\right]+0.01 \mathrm{~mol} / \mathrm{L} \mathrm{K}_{4}\left[\mathrm{Fe}(\mathrm{CN})_{6}\right]$ 溶 液中测试膜电极的反应动力学特征, 发现随着反应 气体中氮气比例增大, 氧化还原峰间隔减小, 电化 学反应的可逆性增强。虽然对掺氮纳米晶和类金刚 石膜的研究取得了一些成果 ${ }^{[10-13]}$, 但在亚微米晶粒 尺度内掺杂情况尚未见报道。另外, 相比于掺硼金 刚石膜, 人们对掺氮金刚石膜的认识仍有不足, 特 别是对其电学和电化学性能及应用方面尚有很多问 题有待研究解决。

本工作采用热阴极直流辉光等离子体化学气相 沉积法制备氮掺杂金刚石膜, 研究掺氮对金刚石膜 结构和电学、电化学性能的影响, 并以硝基苯为目 标, 研究掺氮金刚石电极对硝基苯的电化学催化氧 化降解效果, 探索掺氮金刚石膜在污水处理领域的 应用前景。

\section{1 实验方法}

采用热阴极直流辉光等离子体化学气相沉积 (Hot Cathode Direct Current Plasma Chemical Vapor Deposition, HCDCPCVD) 法制备氮掺杂金刚石 (Nitrogen Doped Diamond, NDD)膜, 祄底选用 $\mathrm{p}$ 型 $\mathrm{Si}$ 片 $\left(10^{-2} \Omega / \mathrm{cm}\right)$, 硅祄底的尺寸约为 $30 \mathrm{~mm} \times$ $30 \mathrm{~mm} \times 0.5 \mathrm{~mm}$ 。沉积时保持工作压力为 $4.5 \times 10^{4} \mathrm{~Pa}$, 放电电压 $750 \mathrm{~V}$ (放电电流约 $8 \mathrm{~A}$ ), 衬底温度 $900^{\circ} \mathrm{C}$, 钽阴极温度 $1100^{\circ} \mathrm{C}$ 。采用 $\mathrm{CH}_{4} / \mathrm{H}_{2} / \mathrm{N}_{2}$ 气体系统, 着 重考查不同氢气氮气流量比对金刚石膜沉积的影 响。保持 $\mathrm{CH}_{4}$ 流量 $5 \mathrm{sccm}$ 不变, $\mathrm{H}_{2}+\mathrm{N}_{2}$ 总流量保 持为 $200 \mathrm{sccm}$, 依次增加 $\mathrm{N}_{2}$ 的流量 $0 、 10 、 20 、 30$ 、 40、 $50 \mathrm{sccm}$, 制备 6 个样品, 编号分别为 $\mathrm{a} \sim \mathrm{f}$, 每个
样品沉积时间 $12 \mathrm{~h}$ 。

采用 S-4800 型场发射扫描电镜(日本日立公司) 观察所制备薄膜的表面形貌、晶粒尺寸等。采用 HP5500 型 Hall 效应测试仪测量样品的导电性能。

采用循环伏安测试 NDD 膜的电化学性质。将 沉积 NDD 薄膜的 $\mathrm{p}$ 型硅片用石蜡涂覆背面, 正面留 出 $1 \mathrm{~cm} \times 1 \mathrm{~cm}$ 的大小, 制成面积为 $1 \mathrm{~cm}^{2}$ 的工作电极, 铂丝和 $\mathrm{Ag} / \mathrm{AgCl}$ 电极分别作为对电极和参比电极, 在 CHI 电化学工作站(CHI660B, 上海辰华仪器有限 公司)进行循环伏安扫描。

硝基苯的阳极电催化降解反应在两电极体系中 进行, 以掺氮金刚石薄膜电极为阳极, Pt 片为阴极, $0.1 \mathrm{~mol} / \mathrm{L} \mathrm{Na}_{2} \mathrm{SO}_{4}$ 溶液为支持电解质, 硝基苯的浓 度为 $0.5 \mathrm{mmol} / \mathrm{L}$, 溶液体积为 $250 \mathrm{~mL}$ 。电解时电位 恒定在 $2.8 \mathrm{~V}$, 电催化氧化过程中恒速搅拌。取样时 间分别为 $0 、 10 、 20 、 30 、 60 、 90 、 120 、 180 、 240$ 、 $300 \mathrm{~min}$, 每次取样 $0.5 \mathrm{~mL}$, 用高效液相色谱仪 (HPLC, UV230+, 大连依利特分析仪器有限公司)分 析硝基苯的浓度。色谱分析实验条件为: Phenomenex ${ }^{\circledR} \mathrm{C} 18$ 反向柱 $(5 \mu \mathrm{m}, 150 \mathrm{~mm} \times 4.6 \mathrm{~mm}), \mathrm{UV}$ 检测 器, 检测波长 $268 \mathrm{~nm}$, 甲醇 : 超纯水 $=85: 15$ 为流 动相, 流速 $1 \mathrm{~mL} / \mathrm{min}$, 进样体积 $20 \mu \mathrm{L}$ 。

反应液的化学需氧量 (COD) 测量: 取溶液 $2.5 \mathrm{~mL}$ 稀释 40 倍后, 按照中华人民共和国国家标准 (GB/T 11892-1989)进行测量 ${ }^{[14]}$ 。

\section{2 结果与讨论}

\subsection{NDD 电极的形貌}

图 1 是不同氮气流量下制备金刚石膜表面的 SEM 照片, 可以看出, 当氮气流量为 $0 \mathrm{sccm}$ 时, 膜 中晶粒较小, 呈圆球形, 粒度约 $200 \mathrm{~nm}$, 晶粒间隙 较小, 排列紧密; 随着氮气的加入及气氛中氮气含 量逐渐增大，膜中金刚石晶粒逐渐长大，晶粒形貌 从球形逐渐展开成尺度约 $500 \mathrm{~nm}$ 的片状, 表面形貌 从菜花状逐渐变为花瓣形团簇状, 团簇尺寸在 $2 \sim 4 \mu \mathrm{m}$, 晶粒间隙较大, 形貌疏松。晶体生长形态 是其内部结构的外在反映, 晶体的各个晶面间的相 对生长速率决定了它的生长形态。气氛中氮气含量 增加后, 氮与碳形成 $\mathrm{CN}$ 基团, 降低了 $\mathrm{C}_{2}$ 基团的浓 度，而 $\mathrm{C}_{2}$ 基团可以促进再形核，增大金刚石的二次 形核率，抑制晶粒长大。 $\mathrm{CN}$ 基团抑制 $\mathrm{C}_{2}$ 基团浓度 

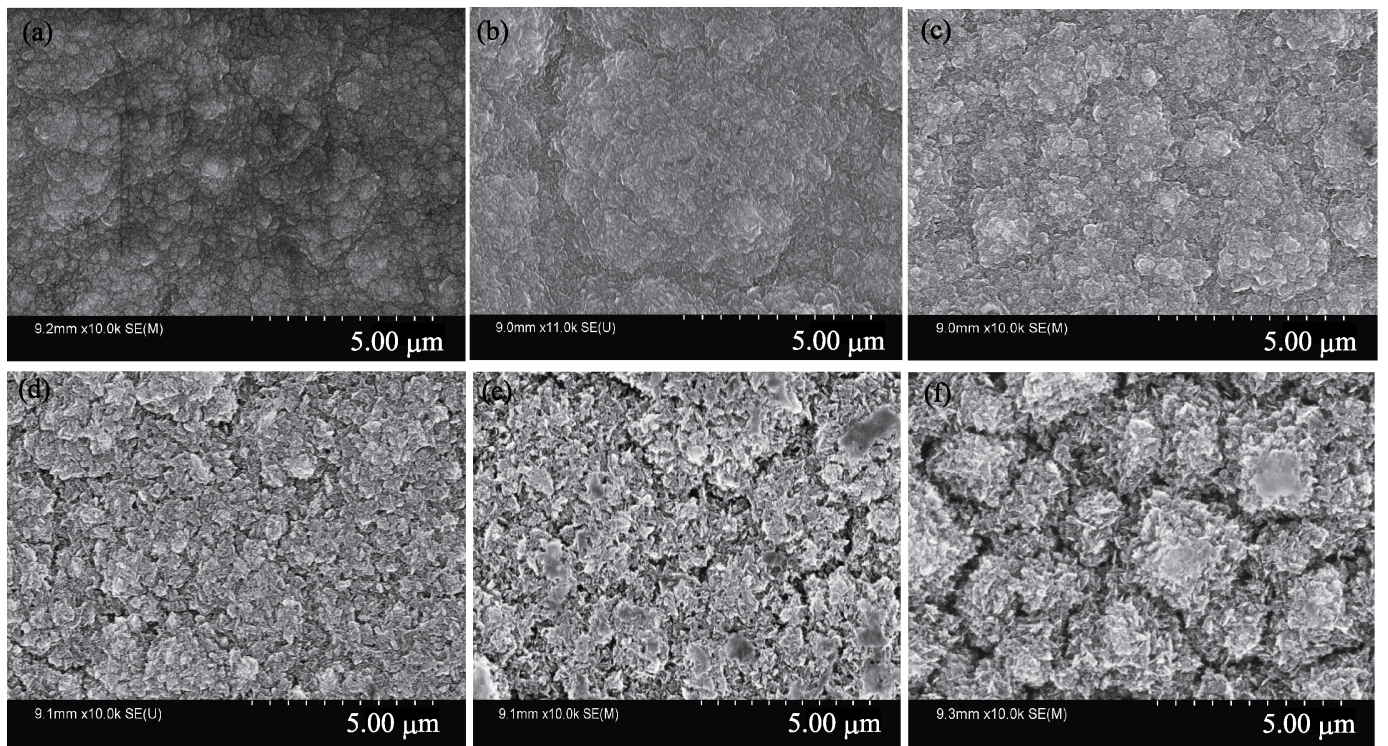

图 1 不同氮气流量下制备的掺氮亚微米晶金刚石膜的 SEM 照片

Fig.1 SEM images of diamond films deposited at different $\mathrm{N}_{2}$ flux rates (a) $0 \mathrm{sccm}$; (b) $10 \mathrm{sccm}$; (c) $20 \mathrm{sccm}$; (d) $30 \mathrm{sccm}$; (e) $40 \mathrm{sccm}$; (f) $50 \mathrm{sccm}$

的结果, 使金刚石晶粒沿某一平面侧向的生长加强, 形成片状结构。

\subsection{NDD 电极的电学性能}

图 2 是 Hall 测量得到样品的电导率随氮气流量 变化曲线, 需要说明的是, 样品 $\mathrm{a}$ 是无氮掺杂样品, 由于该膜的电阻率过高, 低阻件无法测得其电导, 因此图中仅给出样品 $\mathrm{b} \sim \mathrm{f}$ 的测试结果。从图 2 可以 看出, 当氮气流量分别为 $10 、 20 、 30 、 40 、 50 \mathrm{sccm}$ 时, 膜的电导率分别为 $2.444 、 4.885 、 5.091 、 0.647$ 、 $0.012 \mathrm{~S} / \mathrm{cm}$ 。可见, 当氮气流量低于 $30 \mathrm{sccm}$ 时, 膜 的电导率随氮气流量的增大略有提高, 说明掺入氮提 高了膜的导电性能; 当氮气流量继续增大, 则电导 率迅速下降。结合 SEM 分析结果判断可能是氮气流 量增大后, 薄膜晶粒增大且变得疏松, 晶粒间隙增

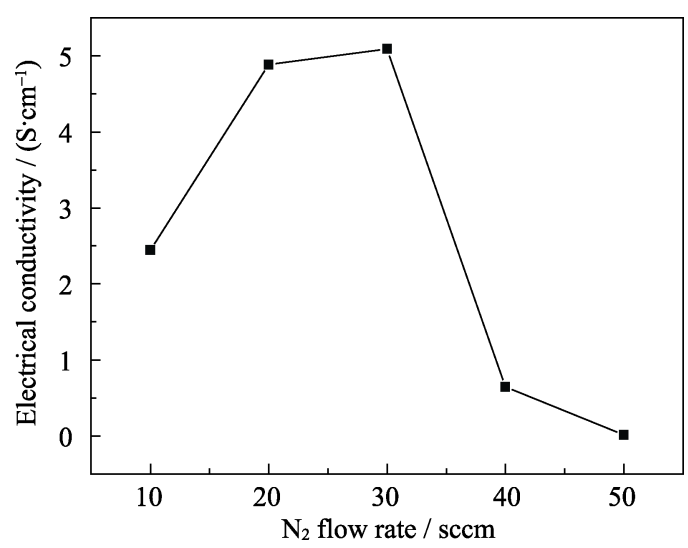

图 2 掺氮亚微米晶金刚石膜电导率随氮气流量变化曲线 Fig. 2 Electrical conductivity of diamond films deposited at different $\mathrm{N}_{2}$ flux rates
大, 导电性变差。从电化学应用方面看, 需要良好导 电性能的金刚石膜，掺氮过高的金刚石膜因结构变 化导致导电性变差, 不利于作为电化学氧化电极应用。

\subsection{NDD 电极的电化学特性}

图 3 是不同氮气流量下沉积 NDD 电极在 $0.1 \mathrm{~mol} / \mathrm{L} \mathrm{Na}_{2} \mathrm{SO}_{4}$ 电解质溶液中的循环伏安曲线, 同样地，样品 $\mathrm{a}$ 由于导电性较差，没有测得结果。从 图 3 可以看出, 所有样品均有较宽的氧化还原电位 窗口, 其中导电率最高的样品 $\mathrm{d}$ 的电位窗口最宽, 背景电流最低, 说明膜的导电性能对其电化学性能 有直接影响。而继续增大氮气流量沉积的样品 $\mathrm{e}$ 和 $\mathrm{f}$ 的背景电流增大, 势窗减小, 电化学性能变差, 与

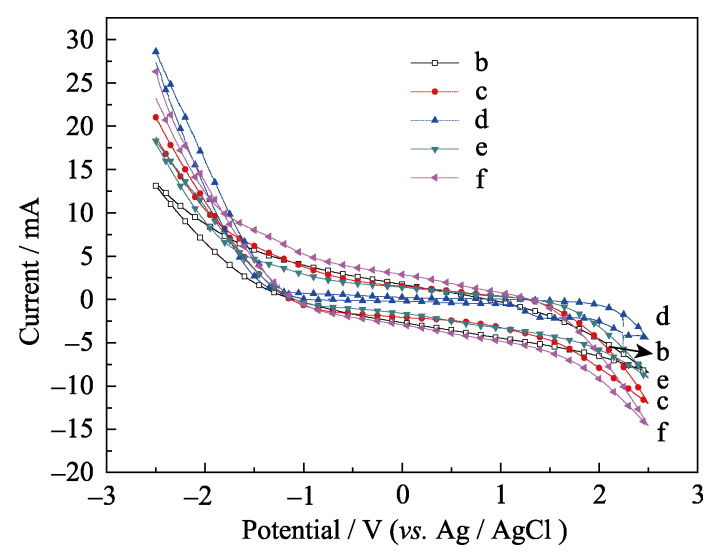

图 3 不同氮流量制备 NDD 电极循环伏安图(0.1 mol/L $\mathrm{Na}_{2} \mathrm{SO}_{4}$; 扫速 $100 \mathrm{mV} \cdot \mathrm{s}^{-1}$ )

Fig. 3 Cyclic voltammogram of the NDD electrodes deposited at different $\mathrm{N}_{2}$ flux rates

(0.1 $\mathrm{mol} / \mathrm{L} \mathrm{Na}_{2} \mathrm{SO}_{4}$; scan rate: $\left.100 \mathrm{mV} \cdot \mathrm{s}^{-1}\right)$

(b) $10 \mathrm{sccm}$; (c) $20 \mathrm{sccm}$; (d) $30 \mathrm{sccm}$; (e) $40 \mathrm{sccm}$; (f) $50 \mathrm{sccm}$ 
其导电率降低的变化过程相一致。图 4 是样品 $\mathrm{c}$ 在 不同电解质溶液中的循环伏安图, 可见无论是在酸性 (0.1 mol/L Na $\left.\mathrm{L}_{2} \mathrm{SO}_{4}-\mathrm{NaHSO}_{4}, \mathrm{pH} 2.2\right)$, 中性 $(0.1 \mathrm{~mol} / \mathrm{L}$ $\left.\mathrm{Na}_{2} \mathrm{SO}_{4}, \quad \mathrm{pH} \quad 7.0\right)$, 还是碱性 $(0.1 \mathrm{~mol} / \mathrm{L}$ $\mathrm{Na}_{2} \mathrm{CO}_{3}-\mathrm{NaHCO}_{3}, \mathrm{pH}$ 9.4)溶液中, 该电极均具有较 宽的电位窗口, 其中在碱性溶液中电位窗口最宽。 在酸性和中性溶液中, 当电位超过 $2.0 \mathrm{~V}$ 后, 有明显 的阳极电流产生, 应是溶液中 $\mathrm{HO}^{-}$离子在 $\mathrm{NDD}$ 电 极上氧化的结果。

\subsection{NDD 电极阳极催化降解硝基苯}

高毒性硝基苯类化合物是危害性较大的环境污 染物, 对生物会产生致癌、致畸和生殖方面等不可 逆转的危害。这种苯环类衍生物化学性质稳定, 不 易开环, 在自然环境下很难自动氧化降解。阳极氧 化技术是处理该类有机污染物的有效途径。而常用 的阳极材料如二氧化铅 ${ }^{[15]}$ 、二氧化锡 ${ }^{[16]}$ 等, 存在着 电流效率低、稳定性差和表面容易被污染等缺点, 实用性较差。金刚石电极由于化学惰性, 利于克服 上述缺点, 成为理想候选材料。

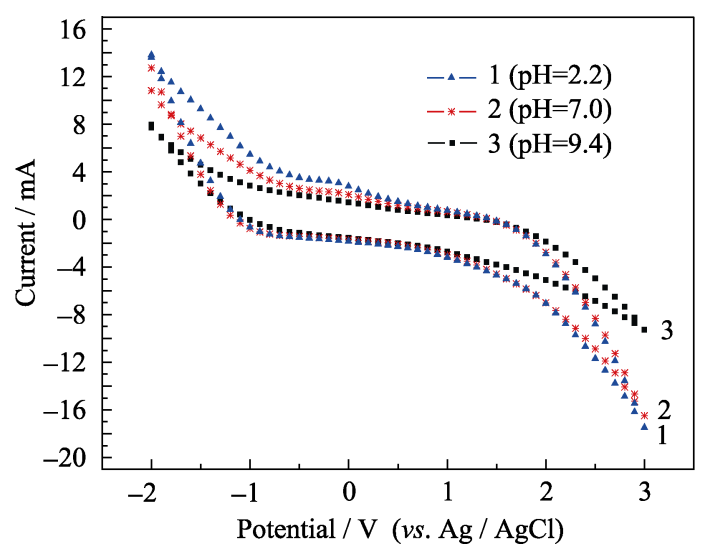

图 4 样品 $\mathrm{c}$ 在不同电解质溶液中的循环伏安图

Fig. 4 Cyclic voltammogram of sample c electrode in different electrolytic solutions at scan rate of $100 \mathrm{mV} / \mathrm{s}$

(1) $0.1 \mathrm{~mol} / \mathrm{L} \mathrm{Na}_{2} \mathrm{SO}_{4}-\mathrm{NaHSO}_{4}, \mathrm{pH} 2.2$; (2) $0.1 \mathrm{~mol} / \mathrm{L} \mathrm{Na}_{2} \mathrm{SO}_{4}, \mathrm{pH} 7.0$; (3) $0.1 \mathrm{~mol} / \mathrm{L} \mathrm{Na}_{2} \mathrm{CO}_{3}-\mathrm{NaHCO}_{3}, \mathrm{pH} 9.4$
实验选择硝基苯 $(\mathrm{NB})$ 为目标物进行阳极氧化降 解, 测试 NDD 电极的电催化活性, 结果如图 5 所 示。从图 5 可以看出, NB 的相对浓度随着电解时间 迅速下降, 特别是伏安性能较好的样品 $\mathrm{d}$ 作电极时, 电解速率最快, $10 \mathrm{~min}$ 内 $\mathrm{NB}$ 的相对浓度就降低到 $60 \%$, 但随后电解速率下降, 至 $180 \mathrm{~min}$ 时 $\mathrm{NB}$ 的相 对浓度仅降低到 $38 \%$, 最终仅降为 $30 \%$, 这可能是 由于降解中间产物吸附到电极表面影响所致。而样 品 $\mathrm{c}$ 对 $\mathrm{NB}$ 的初始降解速度虽低于样品 $\mathrm{d}$ 电极, 但 在 $300 \mathrm{~min}$ 时, NB 相对浓度只有 $6 \%$, 降解效果最佳, 可能是由于样品 $\mathrm{c}$ 表面缺陷较少, 更为平整, 吸附 物较少的缘故。二者比较, 样品 $\mathrm{d}$ 掺氮量略多, 导电 率高，表面凸凹不平，与溶液实际接触面积大，故 此反应初期电解速率快, 但不平整的表面易吸附中 间产物，导致电解效率快速下降。相比之下，掺氮量 高的样品 e 由于晶粒间隙大, 缺陷较多, 导电性差, 对 NB 无论降解速率还是降解效果均低于样品 $\mathrm{c}$ 和 $\mathrm{d}$ 。

苯环类衍生物化学性质稳定, 只有强氧化性的 羟基自由基(HO.)才能使其降解。本反应中, 溶液中 $\mathrm{HO}^{-}$离子在 NDD 电极上氧化生成羟基自由基 $\mathrm{HO}$, $\mathrm{NB}$ 与 HO的反应通常生成一系列羟基化的中间产 物, 如间-硝基苯酚、邻-硝基苯酚、对-硝基苯酚等, 然后在 $\mathrm{O}_{2}$ 分子的参与下发生苯环的开环反应, 生成 各种开环的脂肪烃衍生物, 如已二醛、乙酸和草酸 等, 最终被矿化成 $\mathrm{H}_{2} \mathrm{O} 、 \mathrm{CO}_{2}$ 和 $\mathrm{NO}_{3}^{-[17]}$ 。图 6 分析 了样品 $\mathrm{c}$ 电极降解过程中溶液中 COD 的变化, 结果 显示, 当电解进行至 $300 \mathrm{~min}$ 时, COD 的去除率约 $68 \%$, 而此时的 NB 的降解率达到 $94 \%$ (见图 5 样品 c), 表明 NB 虽被高效降解，但仍有部分中间产物未 被矿化成 $\mathrm{CO}_{2} 、 \mathrm{H}_{2} \mathrm{O}$ 和 $\mathrm{NO}_{3}^{-}$。

\section{3 结论}

采用热阴极直流辉光等离子体化学气相沉积法 制备亚微米晶氮掺杂金刚石膜, 并用 Hall 测试系统
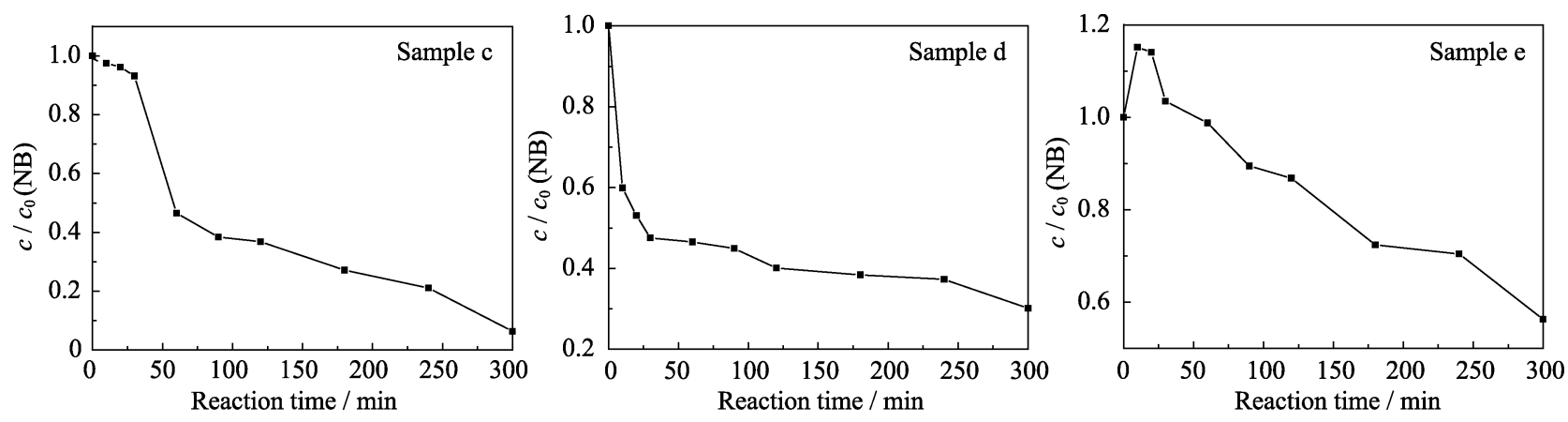

图 5 硝基苯在 NDD 电极(样品 $\mathrm{c}, \mathrm{d}, \mathrm{e}$ )上的阳极催化降解曲线

Fig. 5 Anodically catalyzed degradation curves of NB at NDD electrodes (sample c, d, e) $\left(0.1 \mathrm{~mol} / \mathrm{L} \mathrm{Na}_{2} \mathrm{SO}_{4}\right.$ solution. [NB] $\left.=0.5 \mathrm{mmol} / \mathrm{L}, E=2.8 \mathrm{~V}\right)$ 


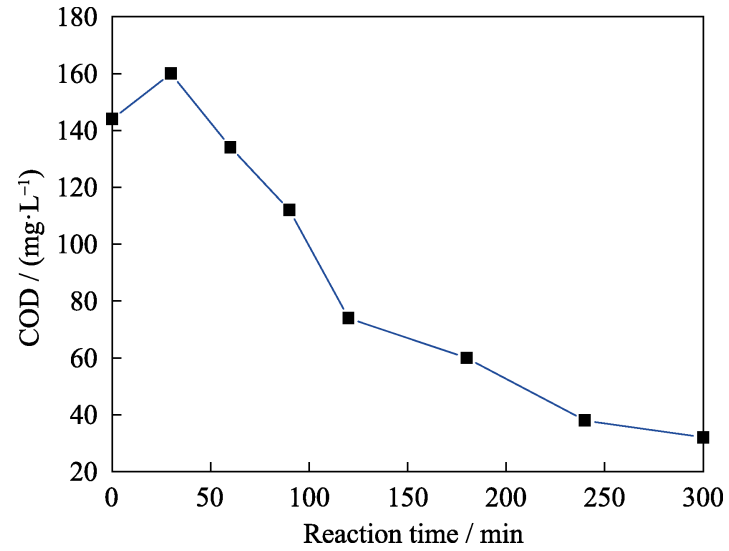

图 6 样品 $\mathrm{c}$ 电解过程中溶液 $\mathrm{COD}$ 的变化曲线

Fig. 6 Anodically catalyzed degradation curve of COD at electrode of sample $\mathrm{c}$

和循环伏安法分别表征氮掺杂金刚石电极的电学和 电化学性能, 以硝基苯为目标污染物测试材料作为 阳极氧化降解的电催化性能。实验结果表明, 当氮 气流量低于 $30 \mathrm{sccm}$ 时, 膜的电导率随氮气流量的 增大略有提高, 表明掺入氮提高了膜的导电性能; 当氮气流量继续增大则电导率迅速下降, 电导率最 大达到 $5.091 \mathrm{~S} / \mathrm{cm}$ 。氮掺杂金刚石电极具有较好的 伏安性能, 在酸性、中性和碱性介质中均具有较宽 的电位窗口和较低的背景电流。在 $0.1 \mathrm{~mol} / \mathrm{L} \mathrm{Na}_{2} \mathrm{SO}_{4}$ 溶液的支持电解质中, 以氮掺杂金刚石为阳极, 铂 片为阴极, 恒电位 $2.8 \mathrm{~V}$ 降解 $0.5 \mathrm{mmol} / \mathrm{L}$ 的硝基苯, 反应时间 $300 \mathrm{~min}$, 硝基苯的降解率 $94 \%$, COD 去除 率约 68\%。可见采用氮掺杂金刚石为阳极，对硝基 苯产生较好的降解效果, 可应用于阳极催化降解该 类有机污染物。

\section{参考文献:}

[1] YU P, XING X, WANG T. Electrochemical performance and charge transfer rate of boron doped diamond with different oxidation extent. Acta Scientiarum Naturalium Universitatis Pekinensis, 2016, 52(5): 911-918.

[2] YUAN K, WANG T, CUI F, et al. Preparation of BDD / Ti Electrode by HFCVD method and its performance in phenol degradation. Surface Technology, 2015, 44(5): 96-101.

[3] WU J L, ZHANG J W, WANG T, et al. Comparative study of benzotriazole electrochemical oxidation at boron-doped diamond and $\mathrm{PbO}_{2}$ anodes. Environmental Science, 2015, 36(7): 2540-2546.

[4] MATSUSHIMA J T, SILVA W M, AZEVEDO A F, et al. The influence of boron content on electroanalytical detection of nitrate using BDD electrodes. Applied Surface Science, 2009, 256: 757762.

[5] ZHAO H X, LIU A P, QIU X, et al. Oxidation behavior of glucose on platinized diamond-like carbon film electrode. Journal of Inorganic Materials, 2010, 25(12): 1325-1329.

[6] HU X J, LI R B, SHEN H S, et al. Hall measurement, FT-IR and EPR for n-type boron and phosphorns co-doped diamond films. Journal of Inorganic Materials, 2004, 19(4): 895-901.

[7] LV J W, FENG Y J, PENG H Y, et al. Influence of B doping on growth characteristic of diamond films prepared by hot cathode DC chemical vapor deposition. Journal of Inorganic Materials, 2009, 24(3): 607-611.

[8] LIU J M, XIA Y B, WANG L J, et al. Effects of process conditions on electric properties of hot-filament CVD diamond films. Journal of Inorganic Materials, 2006, 21(4): 1018-1024.

[9] PLESKOV Y V, KROTOVA M D, SAVELIEV A V, et al. The effects of nitrogenation on the electrochemical properties of nanocrystalline diamond films. Diamond \& Related Materials, 2007, 16: 2114-2117.

[10] PLESKOV Y V, KROTOVA M D, ELKIN V V, et al. n-type nitrogenated nanocrystalline diamond thin-film electrodes: the effect of the nitrogenation on electrochemical properties. Electrochimica Acta, 2007, 52: 5470-5478.

[11] LIU Y M, CHEN S, QUAN X, et al. Nitrogen-doped nanodiamond rod array electrode with superiorperformance for electroreductive debromination of polybrominateddiphenyl ethers. Applied Catalysis B: Environmental, 2014, 154-155: 206-212.

[12] YORIKO T, MASAHIRO F, KOICHI K, et al. Electrochemical properties of $\mathrm{N}$-doped hydrogenated amorphous carbon films fabricated by plasma-enhanced chemical vapor deposition methods. Electrochimica Acta, 2011, 56: 1172-1181.

[13] ZHANG Y R, YOSHIHARA S, SHIRAKASHI T, et al. Electrochemical characteristics of boron-doped, undoped and nitrogendoped diamond films. Diamond \& Related Materials, 2005, 14: 213-219.

[14] GB/T 11892-1989, Water Quality-Determination of Permanganate Index.

[15] ZHOU M H, DAI Q Z, LEI L C, et al. Long life modified lead dioxide anode for organic waste water treatment: electrochemical characteristics and degradation mechanism. Environmental Science and Technology, 2005, 39(1): 363-370.

[16] MONTILLA F, MORALLON E, VAZQUEZ J L. Evaluation of the electrocatalytic activity of antimony-doped tin dioxide anodes toward the oxidation of phenol in aqueous solutions. Journal of the Electrochemical Society, 2005, 152(10): B421-B427.

[17] WANG C T, HUA Y J, WEI J C, et al. Electrocatalytic degradation of nitrobenzene by keggin-type lacunary heteropolyanion $\mathrm{PW}_{11} \mathrm{O}_{39}^{7-}$. Acta Chimica Sinica, 2010, 68(19): 1961-1964. 